\title{
Upper-extremity deep venous thrombosis after whole blood donation: report of three cases from a single blood center
}

\author{
Bruce Newman, ${ }^{1}$ Madhvi Rajpurkar, ${ }^{2}$ Bulent Ozgonenel, ${ }^{2}$ Anup Lal, ${ }^{3}$ and Philip Kuriakose ${ }^{4}$
}

BACKGROUND: There are two upper-extremity deep venous thrombosis (UEDVT) cases after whole blood donation reported in the English medical literature. Three additional UEDVT cases after whole blood donation were reported to our blood center within a 13-month period.

STUDY DESIGN AND METHODS: A case study was done for each case in collaboration with a clinical physician. A description of the donation event, donor demographics, risk factors for thrombosis, treatment, and outcome were described.

RESULTS: A 33-year-old woman and two 17-year-old, first-time-donating men presented with arm pain, swelling, and bruising within hours to 3 days after donation. Two had distal UEDVTs in the basilic or brachial veins, and one had a proximal UEDVT in the subclavian and axillary veins extending into the basilic vein. One donor (woman) had known risk factors for DVT and the other two did not. Anticoagulant therapy was initiated on all patients and was continued for 3, 4, and 9 months. Two donors with the distal UEDVTs recovered completely while the donor with the proximal UEDVT was treated with anticoagulation for 9 months and continued to have a slight residual, nonobstructive thrombosis. The donor was switched to low-dose aspirin prevention. The two donors reported in the literature had complete resolution of thrombosis.

CONCLUSIONS: Four of five donors recovered completely after anticoagulation treatment for UEDVT, including two of three donors in this study. A review of all cases in the medical literature, including 20 recent Australian cases described in an abstract, provides a more complete description of this adverse donation injury.
$\mathrm{U}$ pper-extremity deep venous thrombosis (UEDVT) after whole blood donation is rarely reported. There are two reported cases in the English medical literature. ${ }^{1-3}$ Our institution had three UEDVT cases after whole blood donation within a 13-month period. We report the three cases and also review the two cases reported in the literature. In addition, we are aware of 20 more cases reported in a recent abstract from 4.2 million whole blood donations in Australia, ${ }^{4}$ and we report on the abstract.

\section{MATERIALS AND METHODS}

Three donation events were reported to American Red Cross, SE Michigan Region, by the donors, donors' relatives, or patients' physician. The blood center medical director and a third-party hematologist interviewed the woman donor (Case 1), and hospital and clinic medical records were also obtained for Case 1 . The blood center medical director collaborated with the patients' physicians for Cases 2 and 3. American Red Cross records were obtained for the donations and adverse events.

ABBREVIATIONS: BCP(s) = birth control pill(s); INR = international normalized ratio; UEDVT $=$ upper-extremity deep venous thrombosis.

From the ${ }^{1}$ Medical Office, American Red Cross Blood Services, SE Michigan Region; and the ${ }^{2}$ Division of Hematology/ Oncology, Carman Ann Adams Children's Hospital of Michigan, Wayne State University, Detroit, Michigan; the ${ }^{3}$ Hematology Section, Port Huron Hospital, Port Huron, Michigan; and the ${ }^{4}$ Hematology Section, Henry Ford Hospital, Detroit, Michigan Address reprint requests to: Bruce Newman, MD, Medical Director, American Red Cross Blood Services, Southeastern Michigan Region, 100 E. Mack Avenue, Detroit, MI 48201; e-mail: bruce.newman8@gmail.com.

Received for publication August 27, 2014; revision received November 10, 2014; and accepted November 10, 2014.

doi:10.1111/trf.13034

(C) 2015 AABB

TRANSFUSION 2015;55;1290-1293 
A case study was done for each case in collaboration with the patient's physician. A description of the donation event, donor demographics, risk factors for thrombosis, treatment, and outcome were described.

\section{RESULTS}

\section{Case 1}

A 33-year-old Caucasian woman on birth control pills (BCPs) since 13 years of age donated her fourth whole blood donation of the year in November 2012 from her right arm. A needle adjustment was done because of slow blood flow, and the phlebotomy duration was long, 16 minutes. Three days later the donor telephoned the American Red Cross because of progressive pain, swelling in her right arm, and a small hematoma $(<2 \times 2$ in.). She went to an urgent care center 10 days after her donation because of increasing pain and was referred to a hospital, and a duplex ultrasound showed acute occlusive DVT in her proximal brachial vein that extended to the basilic vein up to the midforearm level. She was treated initially with enoxaparin and bridged to therapeutic warfarin (international normalized ratio [INR], 2-3) for approximately 4 months. Her BCP was changed from an estrogen-derived BCP (drospirenone-ethinyl estradiol, Vestura) to a non-estrogen-containing BCP (northindrone, Micronor). She recovered completely, and there was no recurrence. She had a baby in 2013 and two unremarkable whole blood donations in 2014.

The donor had no previous history of a thrombosis or coagulopathy. Upon investigation, she was found to be positive for being a heterozygote for prothrombin gene variant (G20210A). Factor (F)V Leiden mutation and antiphospholipid antibodies were not detected. She had normal levels for homocysteine and fibrinogen. Her hematologist felt that the phlebotomy issues contributed to the pathogenesis of UEDVT. She was considered to be slightly higher risk for a future thrombosis because of the UEDVT occurrence, having a heterozygous prothrombin gene variant, and taking a BCP, albeit a nonestrogen BCP. Longterm anticoagulation was not indicated, and future pregnancy was considered to be safe.

\section{Case 2}

A 17-year-old, male Caucasian, first-time, high school donor donated whole blood using his left arm in December 2012. The phlebotomy duration was 10 minutes. Within hours, he developed swelling and bruising from the antecubital fossa into the left shoulder. This was associated with mild to moderate pain and discoloration of the skin. The arm partially improved for a day or two and then the swelling and pain progressed further. The father telephoned the American Red Cross 5 days after the donation and was advised to seek urgent care. The donor presented on the sixth day to a hospital emergency room. The entire left upper extremity from the elbow to the deltoid area and into the chest area was swollen with patchy ecchymoses and prominent veins. A venous Doppler ultrasound revealed acute extensive occlusive DVT in the subclavian, axillary, brachial, and basilic veins. The patient was admitted into the hospital for 5 days. He was started on intravenous heparin and transitioned to therapeutic dose warfarin (target INR, 2-3) by the time of hospital discharge. His symptoms resolved rapidly after starting heparin in the hospital, and swelling and pain decreased by 50 $60 \%$ within 24-48 hours after initiation of heparin. His ultrasound at 6 months still showed considerable clot burden-nonocclusive thrombosis in the subclavian vein, occlusive thrombosis in the axillary vein, thrombus in the brachial vein, and superficial thrombus in the cephalic vein. Warfarin was continued for an additional 3 months to obtain further improvement, as he had not been compliant with treatment. A repeat ultrasound at 9 months in October 2013 showed marked improvement, with a small nonocclusive residual thrombus in the subclavian vein. The warfarin was stopped, and the donor was placed on long-term aspirin as a chronic preventative measure against future thrombosis. ${ }^{5}$

The donor had no previous history of a thrombosis or coagulopathy, was a nonsmoker, and was a weightlifter and athlete. Thrombophilia work-up showed that he was heterozygous for MTHFR C677T and the 4G mutation in the tissue plasma activator inhibitor- $1 .{ }^{6}$ Lupus anticoagulant, anti-cardiolipin antibodies, anti-phosphatidylserine antibodies, and FV Leiden were not detected. He had normal activities for antithrombin 3, protein C, and protein S.

\section{Case 3}

A 17-year-old, male Asian Indian, first-time, high school donor donated whole blood using his right arm in December 2013. The phlebotomy duration was 6 minutes. The blood donation was obtained with a good venipuncture, but the donor complained of a tight tourniquet and having to pump his fist throughout the phlebotomy. There was slight pain during the phlebotomy, which the donor did not feel was significant. Over the next several days, he developed pain with arm swelling, bruising, and discoloration in the right upper arm and forearm. At Day 5, physical examination showed an area of erythema on the medial aspect of his right arm and forearm measuring $15 \mathrm{~cm}$ longitudinally and $5 \mathrm{~cm}$ horizontally, with tenderness and increased warmth. There was mild pain over the right biceps muscle and in the antecubital fossa. A venous Doppler ultrasound showed acute occlusive DVT which started at the anterior division of the distal right brachial vein and extended distally. He was prescribed lowmolecular-weight heparin for 3 months. A vascular surgeon evaluated him at 3 weeks, and a repeat ultrasound 


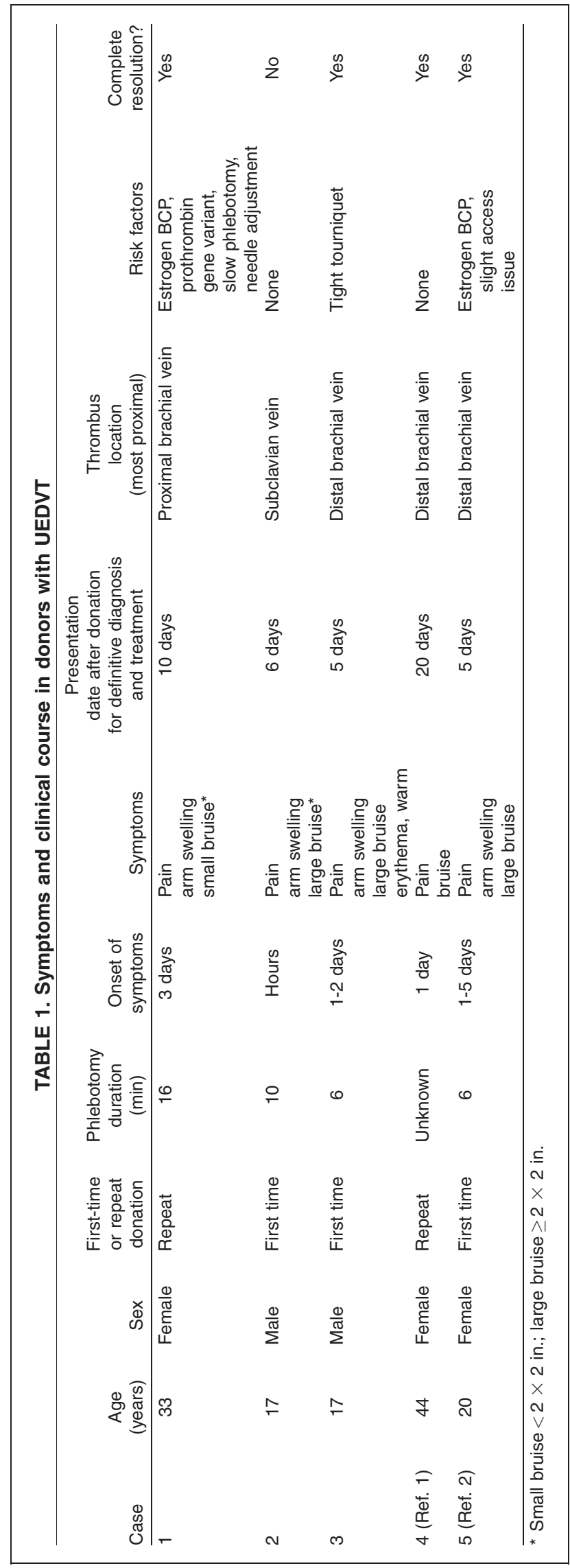

showed the thrombus had resolved. The patient stopped the treatment after 6 weeks on his own. There were no symptomatic recurrent events.

The donor had no previous history of a thrombosis or coagulopathy, was a nonsmoker, and was a weightlifter and cross-country runner. A chest x-ray did not show a cervical rib, and physical examination maneuvers did not reveal a thoracic outlet syndrome. Lupus anticoagulant, cardiolipin antibodies, FV Leiden, and prothrombin G20210A gene variant mutations were not detected. He had normal activities for plasminogen, tissue plasminogen inhibitor, antithrombin, FII, FVIII, protein C, protein S, and von Willebrand antigen.

\section{DISCUSSION}

UEDVTs after whole blood donation are thought to be rare events, with just two reported cases in the English medical literature. ${ }^{3}$ Three reported cases to our institution in a 13-month period suggests that these cases may be underreported. A recent abstract from Australia found 20 confirmed UEDVT cases in 4.2 million collections, for an approximate incidence of one in $211,000{ }^{4}$

The details on the donors in our three cases and on the two reported in the literature are shown in Table 1. Donors most commonly presented with progressive arm pain, arm swelling, and a bruise. Diagnosis of a thrombosis was by ultrasound (four cases) or venogram (one case). One donor had a proximal venous obstruction in the subclavian and axillary veins that extended into the brachial and more distal veins. Four donors had distal venous obstruction in the brachial or basilic or median cubital veins. The five donors were managed with anticoagulants, with four reports stating that it was for 3 to 9 months. Four of the donors had complete resolution of their thrombi, and in one, the thrombus resolved as early as 3 weeks. The fifth donor with thrombosis in the subclavian vein had occlusive residual thrombus at 6 months, but it almost completely resolved by 9 months with better warfarin compliance. The donor was then switched to aspirin.

An evaluation of our three cases, the two cases in the literature, and the 20 cases in Australia based on their abstract provides the most complete description of this donation adverse event. The Australian abstract on 20 cases reports that all donors had confirmed venous thromboses after blood donation and were investigated for thrombotic risk factors. ${ }^{4}$ Data from an abstract should be considered to be preliminary data because the final article may be slightly different. Of the 25 affected donors, 18 (72\%) were women, 13 (52\%) had a phlebotomy issue, 10 $(40 \%)$ were on BCPs, and four (16\%) had a hereditary gene variant associated with increased risk for DVT. These risk factors are not amenable to preventive interventions because the incidence of the event is rare, and any intervention would lead to very large losses of productive 
donors. Six of 25 donors (24\%) had a proximal thrombosis in the subclavian and/or axillary veins. All of the cases were managed with anticoagulant treatment. In general, 24 of 25 donors did well with resolution of the thrombus, no recurrence, and no long-term sequellae. The one exception was one donor that had residual thrombus at the end of treatment period (current report) and was switched to aspirin, but still did well. Another donor had a pulmonary embolus that resolved (abstract). ${ }^{4}$

One of our three donors donated two more times without incident. There are no published data that show recurrence after a subsequent donation. Therefore, it is unclear if such donors should be permanently deferred or not.

An issue is the underreporting of these cases in the English medical literature. Such cases are reported to the blood center but may not be recognized because the blood center does not have a biovigilance system in place, or it does have a biovigilance system in place, but it does not recognize such cases as a separate entity. A third possibility is that the blood center does not realize that such cases should be reported.

UEDVTs are uncommon and can be classified into primary (20\%) and secondary cases $(80 \%) .{ }^{7}$ Primary cases occur because of anatomic abnormalities causing blockage of venous blood flow, repetitive overexertion of the arm usually in athletes, or are idiopathic. Secondary cases of UEDVT occur most commonly after venous cannulation or malignancy. Blood donation would be considered to be a type of venous cannulation albeit transient. Other secondary causes are arm surgery, arm trauma, arm immobilization (plaster cast), and pregnancy. Thrombophilic disorders such as FV Leiden mutation, protein $\mathrm{C}$ or S deficiency, prothrombin gene variants, or antiphospholipid antibodies can increase the risk of venous thrombosis but are present in a minority of patients with UEDVTs. ${ }^{7}$ Patients present with pain, extremity functional impairment, paresthesias, swelling and heaviness of the affected arm, and distended collateral veins. Less than $50 \%$ of the patients with these symptoms have an UEDVT so an ultrasound examination is necessary to confirm the diagnosis. Treatment consists of anticoagulation with initial treatment being either a low-molecular-weight heparin or unfractionated heparin and then bridging to an anti-vitamin $\mathrm{K}$ agent such as warfarin. Treatment is recommended for at least 3 months and INRs should be maintained between 2.0 and 3.0. Possible complications from an UEDVT are postthrombotic syndrome (swelling, pain, heaviness, venous hypertension, and limb fatigue with exertion), pulmonary embolism (uncommon), and recurrent events.

In summary, blood donors that have an UEDVT appear to do well. Their symptoms consist of pain, swelling, and bruising and start within a few hours to a few days after blood donation. The donors generally obtained definitive diagnosis and treatment 5 to 10 days after the donation. Twenty-five percent had a proximal thrombosis in the subclavian or axillary vein and $75 \%$ had a thrombosis more distally in the brachial or basilic or median cubital veins. Twenty-four of 25 donors did well after anticoagulation. None of the donors developed a postthrombotic syndrome or any long-term sequellae, but one donor was placed on long-term aspirin.

\section{CONFLICT OF INTEREST}

The authors have disclosed no conflicts of interest.

\section{REFERENCES}

1. Featherstone T, Bayliss AP. Deep venous thrombosis of the upper extremity—a case report. Angiology 1987;38:793-6.

2. Covin RB, Rich NL, Aysola A. Upper-extremity deep venous thrombosis complicating whole-blood donation. Transfusion 2004;44:586-90.

3. Newman B. Arm complications after manual whole blood donation and their impact. Transfus Med Rev 2013;27:44-9.

4. Bell BM, Pink JM, Keller AJ. Upper limb venous thrombosis complicating blood donation. Blood Transfus 2014;12(Suppl 2):S455(O-02).

5. Becattini C, Agnelli G, Schenone A, et al. Aspirin for preventing the recurrence of venous thromboembolism. N Engl J Med 2012;366:1959-67.

6. Anderson JA, Lim W, Weitz JI. Genetics of coagulation: what the cardiologist needs to know. Can J Cardiol 2013; 29:75-88.

7. Spiezia L, Simioni P. Upper extremity deep vein thrombosis. Intern Emerg Med 2010;5:103-9. 